\title{
Limited-Budget Formation Control for High-Order Linear Swarm Systems with Fix Topologies
}

\author{
Hongtao Dang, ${ }^{1}$ Le Wang $\left(\mathbb{D},{ }^{2}\right.$ Yan Zhang, ${ }^{1}$ and Jianye Yang ${ }^{1}$ \\ ${ }^{1}$ Shannxi Engineering Research Center of Controllable Neutron Source, School of Science, Xijing University, Xi'an 710123, China \\ ${ }^{2}$ High-Tech Institute of Xi'an, Xi'an 710025, China \\ Correspondence should be addressed to Le Wang; wangaz14@163.com
}

Received 31 August 2020; Revised 13 September 2020; Accepted 25 September 2020; Published 8 October 2020

Academic Editor: Ning Cai

Copyright $(92020$ Hongtao Dang et al. This is an open access article distributed under the Creative Commons Attribution License, which permits unrestricted use, distribution, and reproduction in any medium, provided the original work is properly cited.

This paper discusses limited-budget time-varying formation design and analysis problems for a high-order linear swarm system with a fixed communication topology. Firstly, the communication topology among agents is modeled as an undirected and connected graph, and a new formation control protocol with an energy integral term is proposed to realize formation control and to guarantee the practical energy assumption is less than the limited energy budget. Then, by the matrix inequality tool, sufficient conditions for limited-budget formation design and analysis are proposed, respectively, which are scalable and checkable since they are independent of the number of agents of a swarm system and can be transformed into linear matrix inequality constraints. Moreover, an explicit expression of the formation center function is given, which contains the formation function part and the cooperative state part and is not associated with the derivatives of the formation functions. Finally, a numerical simulation is shown to demonstrate the effectiveness of theoretical results.

\section{Introduction}

Recently, many researchers from system and control fields paid their attentions to the distributed coordination of swarm systems, which have potential applications in many aspects, such as flocking algorithm and theory $[1,2]$, synchronization analysis and design [3-11], formation control [12-14], and distributed parallel computation [15-19]. Formation control of a distributed swarm system is inspired by the biological formation behaviors without the superintend node, where it is needed that some specific geometric structure is achieved and maintained by local interactions among all animals. Formation control of distributed swarm systems has extensive application in the coordinated maneuver of multiple androids and coordinated attack of multiple unmanned surface ships. In [20], formation control methods were divided into three classes: the virtual structure method, the behavior-based method, and the synchronization-based method, and it was pointed out that the synchronization-based method is distributed and can overcome some limitations of the virtual structure method and the behavior-based method.
According to the time-varying property of geometric structures, formation is divided into time-invariant geometric structures and time-varying geometric structures. For time-invariant geometric structures, the time derivative of the formation function of a swarm system as a whole is zero; that is, the formation structure is time-invariant once a geometric structure is formed by all agents. For time-varying geometric structures, the time derivative of the formation function of a swarm system as a whole is nonzero, which means that the formation structure is time-varying even if a geometric structure is formed by all agents. By the Nyquist stability criterion, time-invariant formation criteria were proposed in [21]. Du et al. [22] presented time-invariant formation control criteria by using nonlinear formation protocol and applied theoretical results into multiple androids systems. Qin et al. [23] investigated the influences of time delays on the time-invariant formation of swarm systems and gave several sufficient conditions for time-invariant formation control. In [24-26], time-varying formation analysis and design for swarm systems were addressed and some important conclusions for time-varying 
formation were presented. Overall, time-varying formation control is more complex than time-invariant formation control, and some specific formation feasible conditions are needed.

In [27-29], formation control design and analysis problems for swarm systems with different topology structures were investigated, where the influences of the limited energy supply were not dealt with, which is critically important in practical applications. In $[30,31]$, the concept of the guaranteed-cost control was introduced to analyze the impacts of the energy constraint and gave several guaranteed-cost formation criteria. Yu et al. [32] investigated the guaranteed-cost time-varying formation control problem, where the impacts of unknown external disturbances and time delays were considered and the guaranteed-cost timevarying formation criteria were proposed, but the performance cost cannot be determined by their methods. In [30-32], the energy supply of a swarm system as a whole cannot be given previously. However, the whole energy supply is usually limited in practical swarm systems and it is critically important to discuss the influences of the whole energy supply on time-varying formation design and analysis problems. To the best of our knowledge, limitedbudget formation control for high-order linear swarm systems with fixed communication topologies is still open and is not comprehensively addressed and analyzed.

This paper deals with time-varying formation design and analysis for high-order linear swarm systems with the limited energy budget. A formation control protocol is proposed on the basis of the state errors and the formation function errors among neighboring agents, where an energy integral term is introduced to ensure that the whole energy budget is larger than or equal to the practical energy assumption. Furthermore, the dynamics of a swarm system with a fixed communication topology is divided into two parts by the state space decomposition, which can be used to describe the macroscopic motion as a whole and the microcosmic motions between any two agents. By constructing the relationship between the matrix variable and the energy budget, limited-budget formation design and analysis criteria are proposed, respectively, where the scalability property and the checkable property are discussed in detail. Moreover, the formation center function is determined, which involves two independent parts associated with the average value of the time-varying formation functions and the average value of the initial conditions of cooperative states, respectively.

Compared with the recent works on the time-varying formation control, the innovation of this paper is twofold. Firstly, this paper considers the limited-budget time-varying formation control, where the energy budget of the whole swarm system is given and is limited previously. The energy budget was not considered and the practical energy consumption cannot be limited by the energy budget in the time-varying formation as shown in [24-29]. Secondly, the effect of the energy budget to the matrix variable of the matrix inequality conditions is considered and determined, which can introduce the energy constraint into the design of the gain matrix of the time-varying formation control protocol. In contrast, the formation control methods in [24-29] cannot give the specific interaction mechanism for the energy budget.

The main arrangement of this paper is given as follows. The communication topology of a swarm system is modeled and the problem description of limited-budget formation for a swarm system is shown in Section 2. The main results of limited-budget formation are presented in Section 3. A numerical simulation is given to demonstrate the limitedbudget formation criterion in Section 4. Finally, Section 5 summarizes the main conclusions of this paper.

Symbols $R^{n}$ and $R^{n \times n}$, respectively, represent the $n$-dimensional real column vector and the $n$-dimensional real matrix space. $1_{N}$ denotes the $N$-dimensional column vector with all components 1.0 represents the zero vector or zero matrix with compatible dimensions. The symbol $\otimes$ represents the Kronecker product. The notation $Q^{T}=Q>0$ shows that the matrix $Q$ is symmetric and positive definite.

\section{Problem Description}

2.1. Communication Topology Model. For a swarm system with $N$ identical agents, the fixed communication topology is depicted a weighed graph $G=(V(G), E(G))$, where $V(G)=\left\{v_{1}, v_{2}, \cdots, v_{N}\right\}$ is the node set with $v_{k}(k=1,2, \cdots, N)$ representing agent $k$ and $E(G)=\left\{e_{k i}=\left(v_{k}, v_{i}\right)\right\}$ is the edge set with $\left(v_{k}, v_{i}\right)$ denoting the interaction channel from agent $k$ to agent $i$. The notation $N_{k}=\left\{i:\left(v_{i}, v_{k}\right) \in E(G)\right\}$ is used to denote the neighbor set of agent $k$. The symbol $w_{i k}$ denotes the communication weight from agent $k$ to agent $i$, where $w_{i i}=0$ and $w_{i k}=0$ when agent $k$ and agent $i$ are not connected and $w_{i k}>0$ when agent $k$ and agent $i$ are connected. The Laplacian matrix of the communication topology is denoted as $L=\left[l_{i k}\right] \in R^{N \times N}$ with $l_{k k}=\sum_{i \in N_{k}} w_{k i}$ and $l_{i k}=-w_{i k}(k \neq i)$. If the fixed communication topology is connected, then the Laplacian matrix $L$ is symmetric and positive semidefinite; that is, zero is its simple eigenvalue and all its nonzero eigenvalues are positive. More basic concepts and conclusions on graph theory can be found in [33].

2.2. Swarm System Model. The dynamics of each agent is depicted in the following high-order linear system:

$$
\dot{x}_{k}(t)=A x_{k}(t)+B u_{k}(t)
$$

where $k=1,2, \cdots, N, A \in R^{n \times n}$, and $B \in R^{n \times m}$, and $x_{k}(t)$ and $u_{k}(t)$, respectively, denote the cooperative state and the control input. Moreover, a vector-valued function $\eta(t)=$ $\left[\eta_{1}^{T}(t), \eta_{2}^{T}(t), \cdots, \eta_{N}^{T}(t)\right]^{T}$ is used to depict a specific formation structure for swarm system (1) to maintain, where the piecewise continuous differentiable components $\eta_{k}(t)$ are formation functions of agent $k(k=1,2, \cdots, N)$. The formation is time-varying when $\eta(t)$ is time-varying and the formation is fixed when $\eta(t)$ is time-invariant.

In the following, a limited-budget formation control protocol with a fixed communication topology is proposed as follows: 


$$
\left\{\begin{array}{l}
u_{k}(t)=K \sum_{i \in N_{k}} w_{k i}\left(x_{i}(t)-\eta_{i}(t)-x_{k}(t)+\eta_{k}(t)\right), \\
J_{\mathrm{e}}=\sum_{k=1}^{N} \int_{0}^{+\infty} u_{k}^{T}(t) Q u_{k}(t) \mathrm{d} t
\end{array}\right.
$$

where $k=1,2, \cdots, N, K \in R^{m \times n}$, and $Q^{T}=Q>0$, and $J_{\mathrm{e}}$ denotes the practical energy consumption of swarm system (1) as a whole. Let $J_{\max }$ be the maximum energy supply of swarm system (1); that is, the energy budget is limited. The definition of the limited-budget formation control of a swarm system is proposed as follows.

Definition 1. For any given $J_{\max }>0$, swarm system (1) is said to be limited-budget formation achievable by control protocol (2) if there exists a gain matrix $K$ such that $\lim _{t \rightarrow+\infty}\left(x_{k}(t)-\eta_{k}(t)-\eta_{c}(t)\right)=0 \quad(k=1,2, \cdots, N)$ and $J_{\mathrm{e}} \leq J_{\max }$ for any bounded disagreement initial conditions $x_{k}(0)-\eta_{k}(0)(k=1,2, \cdots, N)$, where $\eta_{c}(t)$ is said to be the formation center function.

It can be found from Definition 1 that the formation control is equivalent to the consensus control if $\eta_{k}(t) \equiv 0$ $(k=1,2, \cdots, N)$, which means that the consensus control can be regarded as a special case of the formation control. Besides, the vector $\eta_{k}(t)$ denotes the desired formation shape of the swarm systems and the state of all agents should track $\eta_{k}(t)$ with the formation center function.

The main objective of this paper contains two aspects. The first one is to design the gain matrix $K$ such that swarm system (1) with control protocol (2) achieves limited-budget formation. The second one is to determine an explicit expression of the formation center function.

Remark 1. It should be pointed out that the vector-valued function $\eta(t)$ can be used to design the arbitrary geometric structure with the piecewise continuous differentiable property, but it may be unfeasible for some agent dynamics of a swarm system; that is, the formation achievable property is associated with the mechanism structure of practical dynamic agents in a swarm system. Moreover, the energy consumption of a practical swarm system is limited and control protocol (2) imposes an integral term to guarantee that the actual energy consumption is less than the maximum energy supply. This makes it challenging to construct the relationship between the gain matrix $K$ and the limitedbudget $J_{\max }$, that is, how to design the gain matrix $K$ such that swarm system (1) with control protocol (2) achieves formation under the condition that the maximum energy supply is larger than or equal to the actual energy consumption.

Remark 2. Notice that the practical energy consumption $J_{\mathrm{e}}$ is constructed according to the weight matrix $Q$ and is an integral quadratic associated with $Q$. In the design of the gain matrix, the matrix $Q$ is often considered as a diagonal matrix, whose $k$ th element in the diagonal line denotes the weight to the $k$ th channel of the control protocol. By adjusting the value of the matrix $Q$, the contribution of the control protocol to the practical energy consumption $J_{\mathrm{e}}$ will be changed, and $\mathrm{Q}$ is utilized to determine the matrix variable of the matrix inequality conditions. Moreover, compared with the formation control protocols of [24-29], control protocol (2) introduces the practical energy consumption $J_{\mathrm{e}}$ to save the control energy under the limited-budget constraint; that is, the control gain $K$ should be designed to satisfy that $J_{\mathrm{e}} \leq J_{\max }$ with the weight matrix $\mathrm{Q}$.

\section{Main Results}

Based on the matrix inequality tool, this section gives sufficient conditions for limited-budget formation design and analysis, respectively. Then, an explicit expression of the formation center function is proposed, which contains two parts: the formation function one and the cooperative state one.

For $k=1,2, \cdots, N$, let $\varsigma_{k}(t)=x_{k}(t)-\eta_{k}(t)$, then it can be obtained by (1) and (2) that

$$
\dot{\zeta}_{k}(t)=A\left(\varsigma_{k}(t)+\eta_{k}(t)\right)+B K \sum_{i \in N_{k}} w_{k i}\left(\varsigma_{i}(t)-\varsigma_{k}(t)\right)-\dot{\eta}_{k}(t) .
$$

Let $\varsigma(t)=\left[\varsigma_{1}^{T}(t), \varsigma_{2}^{T}(t), \cdots, \varsigma_{N}^{T}(t)\right]^{T}$, then one can transform swarm system (3) into

$$
\dot{\zeta}(t)=\left(I_{N} \otimes A-L \otimes B K\right) \varsigma(t)+\left(I_{N} \otimes A\right) \eta(t)-\dot{\eta}(t) .
$$

It is assumed that the communication topology of swarm system (1) is undirected and connected, so the Laplacian matrix $L$ is symmetric and positive semidefinite, which owns a simple zero eigenvalue and $N-1$ positive eigenvalues. Hence, one can find an orthonormal matrix $U=\left[1_{N} / \sqrt{N}, \widetilde{U}\right]$ such that $U^{T} L U=\operatorname{diag}\left\{0, \lambda_{2}, \cdots, \lambda_{N}\right\}$, where $0<\lambda_{2} \leq \lambda_{3} \leq \cdots \leq \lambda_{N}$ denote the positive eigenvalues of the Laplacian matrix $L$. One can set that $\widetilde{\varsigma}(t)=\left(U^{T} \otimes I_{n}\right) \varsigma(t)=\left[\widetilde{\varsigma}_{1}^{T}(t), \widetilde{\varsigma}_{2}^{T}(t), \cdots, \widetilde{\varsigma}_{N}^{T}(t)\right]^{T}$, then one can transform swarm system (4) into

$$
\begin{aligned}
& \dot{\tilde{\varsigma}}_{1}(t)=A \widetilde{\varsigma}_{1}(t)+\left(\frac{1}{\sqrt{N}} 1_{N}^{T} \otimes A\right) \eta(t)-\left(\frac{1}{\sqrt{N}} 1_{N}^{T} \otimes I_{n}\right) \dot{\eta}(t), \\
& \dot{\tilde{\varsigma}}_{i}(t)=\left(A-\lambda_{i} B K\right) \widetilde{\varsigma}_{i}(t)+\left(e_{i}^{T} U^{T} \otimes A\right) \eta(t)-\left(e_{i}^{T} U^{T} \otimes I_{n}\right) \dot{\eta}(t),
\end{aligned}
$$

where $e_{i}(i=2,3, \cdots, N)$ represent $N$-dimensional column vectors with the $i$ th part being 1 and zero elsewhere.

The following theorem shows a sufficient condition of limited-budget formation design for swarm system (1) with a fixed communication topology on the basis of the matrix inequality tool, which proposes a design approach of the gain matrix $K$ such that swarm system (1) with control protocol (2) achieves limited-budget formation.

Theorem 1. For any given $J_{\max }>0$ and $W=I_{N}-N^{-1} 1_{N} 1_{N}^{T}$, if $A \eta_{k}(t)-\dot{\eta}_{k}(t)=0(k=1,2, \cdots, N)$ and there exists $P^{T}=$ $P>0$ such that 


$$
\begin{aligned}
& (x(0)-\eta(0))^{T}\left(W \otimes I_{n}\right)(x(0)-\eta(0)) P \leq J_{\max } I_{n}, \\
& A^{T} P+P A-P B B^{T} P+0.25 \lambda_{2}^{-2} \lambda_{N}^{2} P B Q B^{T} P<0,
\end{aligned}
$$

then swarm system (1) is limited-budget formation achievable by control protocol (2) with $K=0.5 \lambda_{2}^{-1} B^{T} P$.

Proof of Theorem 1. Define

$$
\begin{aligned}
& \varsigma_{c}(t) \triangleq\left(U \otimes I_{n}\right)\left[\tilde{\varsigma}_{1}^{T}(t), 0\right]^{T}, \\
& \varsigma_{\bar{c}}(t) \triangleq\left(U \otimes I_{n}\right)\left[0, \widetilde{\varsigma}_{2}^{T}(t), \cdots, \widetilde{\varsigma}_{N}^{T}(t)\right]^{T} .
\end{aligned}
$$

One can find that

$$
\begin{aligned}
& \left(U \otimes I_{n}\right)\left[\widetilde{\varsigma}_{1}^{T}(t), 0\right]^{T}=\frac{1}{\sqrt{N}} 1_{N} \otimes \widetilde{\varsigma}_{1}(t), \\
& \left(U \otimes I_{n}\right)\left[0, \widetilde{\varsigma}_{2}^{T}(t), \cdots, \widetilde{\varsigma}_{N}^{T}(t)\right]^{T}=\sum_{i=2}^{N} U e_{i} \otimes \widetilde{\varsigma}_{i}(t) .
\end{aligned}
$$

Since $\widetilde{\varsigma}(t)=\left(U^{T} \otimes I_{n}\right) \varsigma(t)$, it can be shown that

$$
\varsigma(t)=\left(U \otimes I_{n}\right) \widetilde{\varsigma}(t)
$$

that is,

$$
\varsigma(t)=\varsigma_{c}(t)+\varsigma_{\bar{c}}(t) .
$$

In this case, the transformation matrix $U$ is orthonormal and $\varsigma_{k}(t)=x_{k}(t)-\eta_{k}(t)(k=1,2, \ldots N)$, so it can be found from (8)-(11) that the component $\widetilde{\varsigma}_{1}(t) / \sqrt{N}$ can be used to describe the formation center function $\eta_{c}(t)$ as the time tends to infinity and swarm system (1) with control protocol (2) achieves formation if and only if all the components $\widetilde{\varsigma}_{i}(t)(i=2,3, \cdots, N)$ tend to zero as the time tends to infinity.

In the following, an approach is shown to design the gain matrix $K$ such that all the components $\widetilde{\varsigma}_{i}(t)(i=2,3, \cdots, N)$ tend to zero as the time tends to infinity. Let $P^{T}=P>0$, then one can construct a Lyapunov function candidate as follows:

$$
V_{i}(t)=\widetilde{\varsigma}_{i}^{T}(t) P \widetilde{\varsigma}_{i}(t)(i=2,3, \cdots, N) .
$$

By taking the time derivative of $V_{i}(t)$ along the trajectories of subsystems (6), one can deduce that

$$
\begin{aligned}
\dot{V}_{i}(t)= & \widetilde{\varsigma}_{i}^{T}(t)\left(\left(A-\lambda_{i} B K\right)^{T} P+P\left(A-\lambda_{i} B K\right)\right) \widetilde{\varsigma}_{i}(t) \\
& +2 \widetilde{\varsigma}_{i}^{T}(t) P\left(e_{i}^{T} U^{T} \otimes A\right) \eta(t)-2 \widetilde{\varsigma}_{i}^{T}(t) P\left(e_{i}^{T} U^{T} \otimes I_{n}\right) \dot{\eta}(t) .
\end{aligned}
$$

Let $K=0.5 \lambda_{2}^{-1} B^{T} P$, then it can be found that

$$
\left(A-\lambda_{i} B K\right)^{T} P+P\left(A-\lambda_{i} B K\right) \leq A^{T} P+P A-\lambda_{i} \lambda_{2}^{-1} P B B^{T} P .
$$

Due to $0<\lambda_{2} \leq \lambda_{3} \leq \cdots \leq \lambda_{N}$, it can be shown that $\lambda_{i} \lambda_{2}^{-1} \geq 1(i=2,3, \cdots, N)$. Hence, it can be found by (16) that

$$
A^{T} P+P A-\lambda_{i} \lambda_{2}^{-1} P B B^{T} P \leq A^{T} P+P A-P B B^{T} P .
$$

$$
\begin{aligned}
\Pi(t)= & {\left[\left(A \eta_{1}(t)-\dot{\eta}_{1}(t)\right)^{T},\left(A \eta_{2}(t)-\dot{\eta}_{2}(t)\right)^{T}, \cdots,\right.} \\
& \left.\left(A \eta_{N}(t)-\dot{\eta}_{N}(t)\right)^{T}\right]^{T} .
\end{aligned}
$$

Since

$$
e_{i}^{T} U^{T} \otimes A=\left(e_{i}^{T} U^{T} \otimes I_{n}\right)\left(I_{N} \otimes A\right),
$$

it can be derived by (18) that

$$
\left(e_{i}^{T} U^{T} \otimes A\right) \eta(t)-\left(e_{i}^{T} U^{T} \otimes I_{n}\right) \dot{\eta}(t)=\left(e_{i}^{T} U^{T} \otimes I_{n}\right) \Pi(t) .
$$

If

$$
A \eta_{k}(t)-\dot{\eta}_{k}(t)=0(k=1,2, \cdots, N),
$$

then one can show by (18) that

$$
\Pi(t)=0 .
$$

In this case, since $U^{T} \otimes I_{n}$ is invertible, one can find that

$$
\left(e_{i}^{T} U^{T} \otimes A\right) \eta(t)-\left(e_{i}^{T} U^{T} \otimes I_{n}\right) \dot{\eta}(t)=0 .
$$

Thus, if $A^{T} P+P A-P B B^{T} P<0$, then $\lim _{t \rightarrow+\infty} \widetilde{\varsigma}_{i}(t)=0$ $(i=2,3, \cdots, N)$ by (15), (17), and (23). Hence, swarm system (1) with a fixed communication topology is formation achievable by control protocol (2) with $K=0.5 \lambda_{2}^{-1} B^{T} P$.

Finally, the influences of the limited budget are analyzed. By (2), one can find that

$$
J_{\mathrm{e}}=\int_{0}^{+\infty} \varsigma^{T}(t)\left(L^{2} \otimes K^{T} \mathrm{Q} K\right) \varsigma(t) \mathrm{d} t .
$$

By $K=0.5 \lambda_{2}^{-1} B^{T} P$ and $\widetilde{\varsigma}(t)=\left(U^{T} \otimes I_{n}\right) \varsigma(t)$, it can be deduced by (27) that

$$
\varsigma^{T}(t)\left(L^{2} \otimes K^{T} Q K\right) \varsigma(t) \leq 0.25 \lambda_{2}^{-2} \sum_{i=2}^{N} \lambda_{i}^{2} \widetilde{\varsigma}_{i}^{T}(t) P B Q B^{T} P \widetilde{\varsigma}_{i}(t) .
$$

Let $\phi \geq 0$, then one has

$$
\begin{aligned}
J_{\mathrm{e}}^{\phi} & =\int_{0}^{\phi} \varsigma^{T}(t)\left(L^{2} \otimes K^{T} \mathrm{Q} K\right) \varsigma(t) \mathrm{d} t \\
& \leq 0.25 \lambda_{2}^{-2} \sum_{i=2}^{N} \int_{0}^{\phi} \lambda_{i}^{2} \widetilde{\varsigma}_{i}^{T}(t) P B Q B^{T} P \widetilde{\varsigma}_{i}(t) \mathrm{d} t .
\end{aligned}
$$

By

$$
\int_{0}^{\phi} \dot{V}_{i}(t) \mathrm{d} t=V_{i}(\phi)-V_{i}(0),
$$

it can be derived from (27)-(29) that

$$
\begin{aligned}
J_{\mathrm{e}}^{\phi} \leq & \sum_{i=2}^{N} \int_{0}^{\phi}\left(\left(\dot{V}_{i}(t)+0.25 \lambda_{2}^{-2} \lambda_{i}^{2} \widetilde{\varsigma}_{i}^{T}(t) P B Q B^{T} P \widetilde{\varsigma}_{i}(t)\right) \mathrm{d} t\right. \\
& \left.-V_{i}(\phi)\right)+\sum_{i=2}^{N} V_{i}(0) .
\end{aligned}
$$

One can set that 
Since $\lambda_{N}$ is the maximum eigenvalue of the Laplacian matrix $L$ and $\lambda_{i} \lambda_{2}^{-1} \geq 1(i=2,3, \cdots, N)$, one can derive by (31) that

$$
\begin{aligned}
J_{\mathrm{e}}^{\phi} \leq & \sum_{i=2}^{N} \int_{0}^{\phi}\left(\left(\dot{V}_{i}(t)+0.25 \lambda_{2}^{-2} \lambda_{N}^{2} \widetilde{\varsigma}_{i}^{T}(t) P B Q B^{T} P \widetilde{\varsigma}_{i}(t)\right) \mathrm{d} t\right. \\
& \left.-V_{i}(\phi)\right)+\sum_{i=2}^{N} V_{i}(0) .
\end{aligned}
$$

\section{If}

$$
A^{T} P+P A-P B B^{T} P+0.25 \lambda_{2}^{-2} \lambda_{N}^{2} P B Q B^{T} P<0,
$$

then all the components $\widetilde{\varsigma}_{i}(t)(i=2,3, \cdots, N)$ tend to zero as the time $t$ tends to infinity, and $V_{i}(\phi)(i=2,3, \cdots, N)$ tend to zero as the parameter $\phi$ the time tends to infinity; that is, $\lim _{t \longrightarrow+\infty} \widetilde{\varsigma}_{i}(t)=0$ and $\lim _{\phi \rightarrow+\infty} V_{i}(\phi)=0(i=2,3, \cdots, N)$. Thus, it can be found by (27) and (30) that

$$
J_{\mathrm{e}} \leq \sum_{i=2}^{N} \widetilde{\varsigma}_{i}^{T}(0) P \widetilde{\varsigma}_{i}(0)
$$

Due to

$$
\widetilde{\varsigma}_{i}(0)=\left(e_{i}^{T} U^{T} \otimes I_{n}\right) \varsigma(0)(i=2,3, \cdots, N),
$$

one can derive that

$$
\begin{aligned}
\sum_{i=2}^{N} \widetilde{\varsigma}_{i}^{T}(0) P \widetilde{\varsigma}_{i}(0)= & \varsigma^{T}(0)\left(\left[\begin{array}{c}
e_{2}^{T} U^{T} \\
\vdots \\
e_{N}^{T} U^{T}
\end{array}\right]^{T} \otimes I_{n}\right) \\
& \cdot\left(I_{N} \otimes P\right)\left(\left[\begin{array}{c}
e_{2}^{T} U^{T} \\
\vdots \\
e_{N}^{T} U^{T}
\end{array}\right] \otimes I_{n}\right) \varsigma(0) .
\end{aligned}
$$

Since

$$
U=\left[1_{N} / \sqrt{N}, \widetilde{U}\right],
$$

it can be shown that

$$
U U^{T}=I_{N}=\frac{1}{N} 1_{N} 1_{N}^{T}+\widetilde{U} \widetilde{U}^{T},
$$

that is,

$$
\widetilde{U} \widetilde{U}^{T}=I_{N}-\frac{1}{N} 1_{N} 1_{N}^{T} .
$$

Since $\left[U e_{2}, U e_{3}, \cdots, U e_{N}\right]=\widetilde{U}$, from (34)-(36), it can be found that

$$
J_{\mathrm{e}} \leq \varsigma^{T}(0)(W \otimes P) \varsigma(0),
$$

where $W=I_{N}-N^{-1} 1_{N} 1_{N}^{T}$. It is assumed that $x_{k}(0)-f_{k}(0)$ where $(k=1,2, \cdots, N)$ are disagreement, so there always exists some $\widetilde{\varsigma}_{i}(0) \neq 0(i \in\{2,3, \cdots, N\})$. Hence, it can be obtained that

$$
\varsigma^{T}(0)\left(W \otimes I_{n}\right) \varsigma(0)=\sum_{i=2}^{N} \widetilde{\varsigma}_{i}^{T}(0) \widetilde{\varsigma}_{i}(0)>0 .
$$

In this case, there exists a positive scalar $\xi$ such that

$$
J_{\max }=\varsigma^{T}(0)\left(W \otimes \xi I_{n}\right) \varsigma(0) .
$$

The matrix $W$ has a simple zero eigenvalue and $N-1$ nonzero eigenvalues are positive, so it can be derived by (37) and (39) that $P \leq \xi I_{n}$ can guarantee that $J_{\mathrm{e}} \leq J_{\max }$, which means that

$$
(x(0)-\eta(0))^{T}\left(W \otimes I_{n}\right)(x(0)-\eta(0)) P \leq J_{\max } I_{n} .
$$

Thus, the Proof of Theorem 1 is completed.

In Theorem 1, a limited-budget formation design criterion is proposed, which gives a design approach of the gain matrix $K$ of control protocol (2) to make swarm system (1) achieve limited-budget formation. If the gain matrix $K$ is given, then the following theorem shows a limited-budget formation analysis criterion, which can be obtained directly according to the Proof of Theorem 1.

Theorem 2. For any given $J_{\max }>0, K$ and $W=I_{N}-N^{-1} 1_{N} 1_{N}^{T}$, swarm system (1) with control protocol (2) achieves limited-budget formation if $A \eta_{k}(t)-\dot{\eta}_{k}(t)=0$ $(k=1,2, \cdots, N)$ and there exists $P^{T}=P>0$ such that

$$
\begin{gathered}
(x(0)-\eta(0))^{T}\left(W \otimes I_{n}\right)(x(0)-\eta(0)) P \leq J_{\max } I_{n}, \quad \\
A^{T} P+P A-\lambda_{i} P B K-\lambda_{i} K^{T} B^{T} P+\lambda_{N}^{2} K^{T} Q K<0(i=2,3, \cdots, N) .
\end{gathered}
$$

There exist two difficulties to check the matrix inequalities in Theorem 2. The first one is that it is not scalable since the number of constraint conditions $A^{T} P+P A-\lambda_{i}$ $P B K-\lambda_{i} K^{T} B^{T} P+\lambda_{N}^{2} K^{T} Q K<0(i=2,3, \cdots, N)$ increases as the number of agents increases. The second one is that the term $\lambda_{N}^{2} K^{T} Q K$ makes matrix inequalities $A^{T} P+P A-$ $\lambda_{i} P B K-\lambda_{i} K^{T} B^{T} P+\lambda_{N}^{2} K^{T} Q K<0(i=2,3, \cdots, N)$ not linear, which are difficult to be checked. By the convex feature of linear matrix inequalities and the Schur lemma in [34], the following theorem can be obtained on the basis of Theorem 2 , which is scalable and linear and can be checked by the feasp solver in the Matlab's LMI toolbox given in [35] and will bring no conservatism from Theorem 2 to Theorem 3 .

Theorem 3. For any given $J_{\max }>0, K$ and $W=I_{N}-N^{-1} 1_{N} 1_{N}^{T}$, swarm system (1) with control protocol (2) achieves limited-budget formation if $A \eta_{k}(t)-\dot{\eta}_{k}(t)=0$ $(k=1,2, \cdots, N)$ and there exists $P^{T}=P>0$ such that

$$
\begin{gathered}
(x(0)-\eta(0))^{T}\left(W \otimes I_{n}\right)(x(0)-\eta(0)) P \leq J_{\max } I_{n}, \quad(43) \\
{\left[\begin{array}{cc}
A^{T} P+P A-\lambda_{i} P B K-\lambda_{i} K^{T} B^{T} P & \lambda_{N} K^{T} Q \\
\lambda_{N} Q K & -Q
\end{array}\right]<0(i=2, N) .}
\end{gathered}
$$

Remark 3. Intuitively speaking, by designing piecewise continuous differentiable $\eta_{k}(t)(k=1,2, \cdots, N)$, arbitrarily required formation structures for swarm system (1) with control protocol (2) to maintain can be obtained. However, 
the achievability of a geometric structure is dependent on the structure property of a swarm system; that is, the achievability is closely related to the system matrix $A$. If the formation functions $\eta_{k}(t)(k=1,2, \cdots, N)$ are not timevarying, that is, $\eta_{k}(t)(k=1,2, \cdots, N)$ are constant, then the condition $A \eta_{k}(t)=0(k=1,2, \cdots, N)$ is needed for swarm system (1) with control protocol (2) to achieve time-variant formation structures as shown in [15]. If the formation functions $\eta_{k}(t)(k=1,2, \cdots, N)$ are time-varying, then Theorem 1 requires that the condition $A \eta_{k}(t)-\dot{\eta}_{k}(t)=0$ $(k=1,2, \cdots, N)$ for swarm system (1) with control protocol (2) to achieve time-varying formation structures. The literature studies $[25,26]$ showed some similar feasible conditions for swarm systems to achieve time-varying formation structures, where feasible conditions are different from the one in Theorem 1 since the limited budget was not taken into consideration.

Remark 4. Because the whole energy budget must be limited in practical engineering swarm systems, it is critically important to analyze the impacts of the limited budget for swarm system (1) with control protocol (2) to achieve specific formation; that is, swarm system (1) with control protocol (2) can obtain and maintain specific geometric structures under the condition that the whole energy consumption is restricted. Note that the condition $(x(0)-\eta(0))^{T}\left(W \otimes I_{n}\right)(x(0)-\eta(0)) P \leq J_{\max } I_{n}$ introduces the maximum energy supply $J_{\max }$ into the gain matrix design, where the initial condition $x(0)-\eta(0)$ and the interaction relationship matrix $W$ are used. The initial condition is often available in practical applications and the interaction relationship matrix shows the impact of the topology. By the structure property of the transformation matrix $U$, Theorem 1 proposes an approach to construct the interaction relationship between the limited budget and the matrix variable, which introduces the limited budget into the formation criterion. It should be pointed out that the interaction relationship matrix $W=I_{N}-N^{-1} 1_{N} 1_{N}^{T}$ can be regarded as the Laplacian matrix of a complete graph with all edge weights being $N^{-1}$. In this case, the interaction relationship matrix has a simple zero eigenvalue and $N-1$ identical nonzero eigenvalues. Moreover, the sufficient conditions in Theorem 1 for swarm system (1) with control protocol (2) to achieve limited-budget formation are scalable since they are independent of the number of agents and the two eigenvalues $\lambda_{2}$ and $\lambda_{N}$ of the Laplacian matrix can be estimated by the approaches in $[36,37]$.

By the analysis of Theorem 1 , the component $\widetilde{\varsigma}_{1}(t) / \sqrt{N}$ can be applied to depict the formation center function $\eta_{c}(t)$ as the time tends to infinity, which is associated with subsystem (5). The following theorem shows a method to give an explicit expression of the formation center function.

Theorem 4. If swarm system (1) with control protocol (2) achieves limited-budget formation, then the formation center function satisfies that $\lim _{t \rightarrow \longrightarrow^{+}}+\infty\left(\eta_{c}(t)-\eta_{c f}(t)-\eta_{c x}(t)\right)=0$, where $\eta_{c f}(t)=-N^{-1} \sum_{k=1}^{N} \eta_{k}(t)$ and $\eta_{c x}(t)=N^{-1}$ $e^{A t} \sum_{k=1}^{N} x_{k}(0)$.
Proof of Theorem 4. Due to

$$
\begin{aligned}
\widetilde{\varsigma}(0) & =\left(U^{T} \otimes I_{n}\right) \varsigma(0), \\
\varsigma_{k}(0) & =x_{k}(0)-\eta_{k}(0)(k=1,2, \cdots, N),
\end{aligned}
$$

it can be shown that

$$
\widetilde{\varsigma}_{1}(0)=\left(e_{1}^{T} U^{T} \otimes I_{n}\right) \varsigma(0)=\frac{1}{\sqrt{N}}\left(\sum_{k=1}^{N} x_{k}(0)-\sum_{k=1}^{N} \eta_{k}(0)\right) \text {. }
$$

Furthermore, the dynamic response of subsystem (5) introduced by initial conditions is that

$$
\varsigma_{0}(t)=e^{A t} \widetilde{\varsigma}_{1}(0) .
$$

By (47) and (48), one can see that

$$
\varsigma_{0}(t)=\frac{1}{\sqrt{N}} e^{A t}\left(\sum_{k=1}^{N} x_{k}(0)-\sum_{k=1}^{N} \eta_{k}(0)\right) .
$$

Moreover, the dynamic response of subsystem (5) introduced by the formation function $\eta(t)$ is that

$$
\varsigma_{\eta}(t)=\frac{1}{\sqrt{N}} \sum_{k=1}^{N} \int_{0}^{t} e^{A(t-\varphi)} A \eta_{k}(\varphi) \mathrm{d} \varphi .
$$

In particular, the dynamic response of subsystem (5) introduced by the derivative of the formation function $\dot{\eta}(t)$ is that

$$
\begin{aligned}
\varsigma_{\dot{\eta}}(t)= & -\frac{1}{\sqrt{N}} \int_{0}^{t} e^{A(t-\varphi)}\left(1_{N}^{T} \otimes I_{n}\right) \dot{\eta}(\varphi) \mathrm{d} \varphi \\
= & -\frac{1}{\sqrt{N}}\left(\sum_{k=1}^{N} \eta_{k}(t)+e^{A t} \sum_{k=1}^{N} \eta_{k}(0)\right) \\
& -\frac{1}{\sqrt{N}} \sum_{k=1}^{N} \int_{0}^{t} A e^{A(t-\varphi)} \eta_{k}(\varphi) \mathrm{d} \varphi .
\end{aligned}
$$

The component $\widetilde{\varsigma}_{1}(t) / \sqrt{N}$ can be applied to depict the formation center function $\eta_{c}(t)$ as the time tends to infinity, so from (50) and (51), one can show that

$$
\lim _{t \rightarrow+\infty}\left(\eta_{c}(t)-\eta_{c f}(t)-\eta_{c x}(t)\right)=0,
$$

where

$$
\begin{aligned}
& \eta_{c f}(t)=-\frac{1}{N} \sum_{k=1}^{N} \eta_{k}(t), \\
& \eta_{c x}(t)=\frac{1}{N} e^{A t} \sum_{k=1}^{N} x_{k}(0) .
\end{aligned}
$$

Thus, the Proof of Theorem 4 is completed.

Remark 5. Limited-budget formation motions of a swarm system with a fixed communication topology contain two parts. The first part is the macroscopic motion as a whole and 
the second part is the microcosmic motions between any two agents. Limited-budget formation is obtained if and only if the microcosmic motions between any two agents are asymptotically stable. If limited-budget formation is achieved, then all agents in a swarm system move along the trajectories of the formation center function, which can be used to depict the macroscopic motion as a whole. From Theorem 4, the formation center function involves two parts, which are associated with the average value of the time-varying formation functions and the average value of the initial conditions of cooperative states, which are called the formation function part and the cooperative state part, but it is not associated with the derivatives of the formation functions; that is, the variance of the formation structure does not impact the macroscopic motion as a whole. In particular, according to the explicit expression of the formation center function in Theorem 4, the limited budget of energy assumptions does not also influence the macroscopic motion as a whole.

\section{Numerical Simulations}

This section provides the numerical simulation to demonstrate the correctness of our main conclusions about limitedbudget formation control for swarm systems with fixed communication topologies.

Consider a swarm system with five agents, where the dynamics of each agent is modeled by (1) with

$$
\begin{aligned}
& A=\left[\begin{array}{ccc}
0 & 0 & -1 \\
0 & 0 & 1 \\
-1 & -2 & 0
\end{array}\right], \\
& B=\left[\begin{array}{c}
1 \\
-0.5 \\
-1
\end{array}\right] .
\end{aligned}
$$

The communication topology of this swarm system is depicted in Figure 1, where the edge weight is set to be $0-1$; that is, the weight of the connected edge is one and the weight of the unconnected edge is zero. The initial conditions of the cooperative states are presented as follows:

$$
\begin{aligned}
& x_{1}(0)=[3.2,1.8,-2.1]^{T}, \\
& x_{2}(0)=[4.4,-2.7,0.2]^{T}, \\
& x_{3}(0)=[-3.3,1.5,6.1]^{T}, \\
& x_{4}(0)=[-0.8,2.9,1.4]^{T}, \\
& x_{5}(0)=[-1.1,3.9,-3.8]^{T} .
\end{aligned}
$$

The formation functions are chosen as follows:

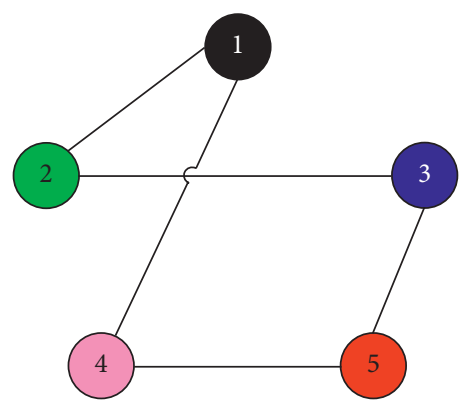

FIgURE 1: Communication topology.

$$
\eta_{k}(t)=\left[\begin{array}{c}
\sin \left(t+\frac{2(k-1) \pi}{5}\right) \\
-\sin \left(t+\frac{2(k-1) \pi}{5}\right) \\
-\cos \left(t+\frac{2(k-1) \pi}{5}\right)
\end{array}\right](k=1,2, \cdots, 5) .
$$

One can find that the formation functions $\eta_{k}(t)(k=$ $1,2, \cdots, 5)$ satisfy the feasibility conditions $A \eta_{k}(t)-\dot{\eta}_{k}(t)=$ $0(k=1,2, \cdots, 5)$ in Theorem 1. Let $Q=0.1$ and $J_{\max }=1000$. By the feasp solver in the Matlab's LMI toolbox, it can be found from Theorem 1 that

$$
P=\left[\begin{array}{lll}
7.0480 & 0.9924 & 0.0699 \\
0.9924 & 8.4394 & 1.2820 \\
0.0699 & 1.2820 & 3.3207
\end{array}\right]
$$

In this case, the gain matrix is $K=[2.3452,-1.6315,-1.4081]$.

In Figure 2, the trajectories of $\varsigma_{k}(t)(k=1,2, \cdots, 5)$ for this swarm system are presented, where the curves depicted by blue star markers represent the trajectories of the formation center function. Figure 3 shows the state snapshots of five agents and the formation center at $t=0 \mathrm{~s}, t=8 \mathrm{~s}, t=9 \mathrm{~s}$, and $t=10 \mathrm{~s}$, where five agents are depicted by black circles, green plusses, blue asterisks, pink x-marks, and red hexagrams, orderly. By Figure 3, one can find that all the agents achieve a time-varying pentagon around the formation center at different times.

The formation center of this swam system counterclockwise rotates about 1.62 cycles as shown in Figure 4, where the initial state is denoted by a red pentacle and the final state is represented by a black pentacle.

Figure 5 shows that the practical energy consumption $J_{\mathrm{e}}(t)$ converges to a finite value less than $J_{\max }$. From Figures 2-5, one can see that this swam system achieves the desired pentagonal formation in the form of the circular movement and the limited budget is guaranteed. 


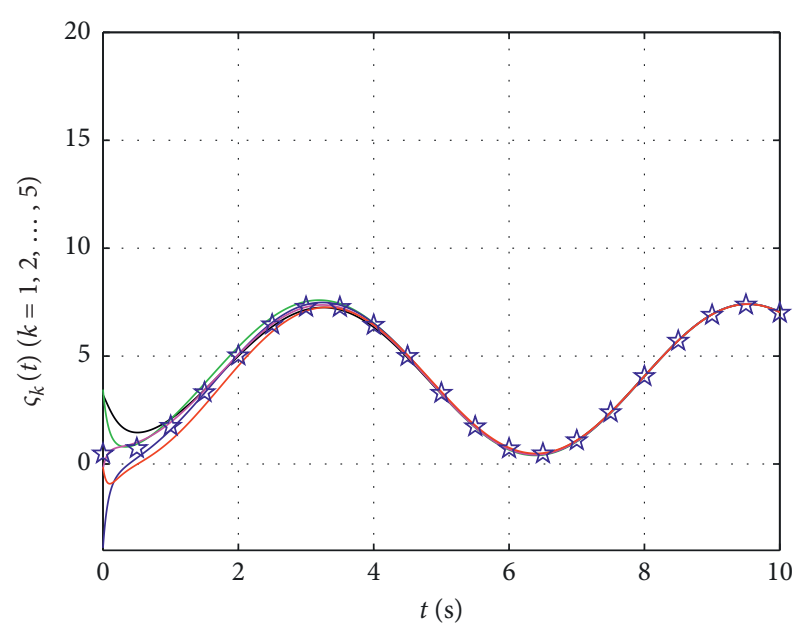

$-\varsigma_{1}(t)$
$-\varsigma_{2}(t)$
$-\varsigma_{3}(t)$

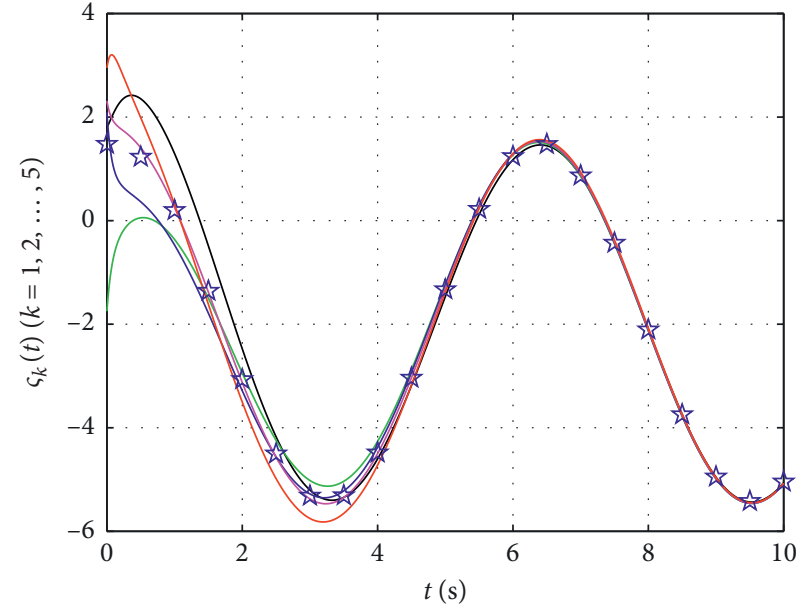

$-\varsigma_{1}(t)$
$-\varsigma_{2}(t)$
$-\varsigma_{3}(t)$

$-\varsigma_{4}(t)$$$
-\varsigma_{5}(t)
$$

(b)

(a)
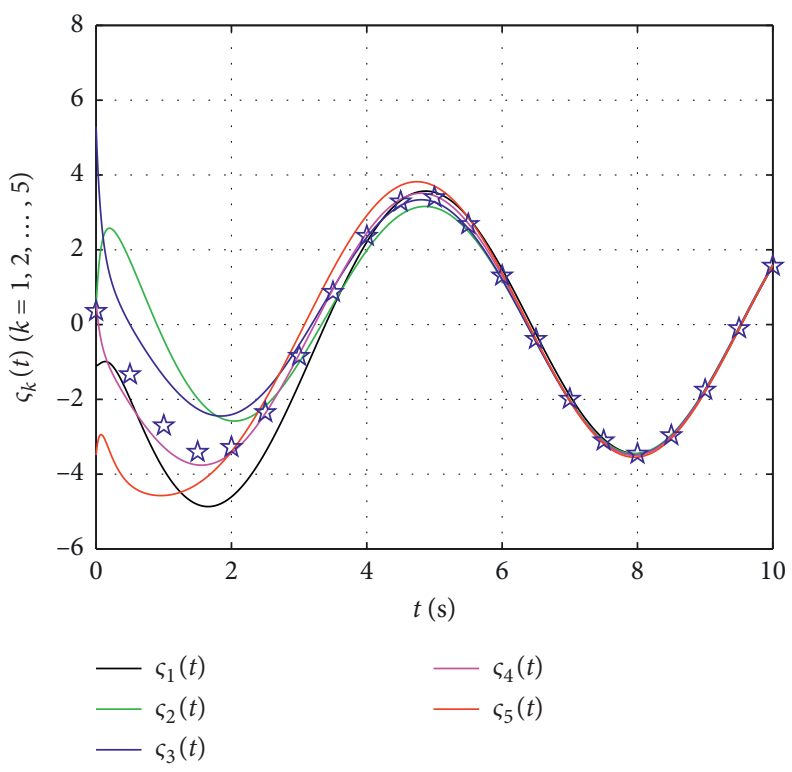

(c)

Figure 2: Trajectories of $\varsigma_{k}(t)(k=1,2, \cdots, 5)$.

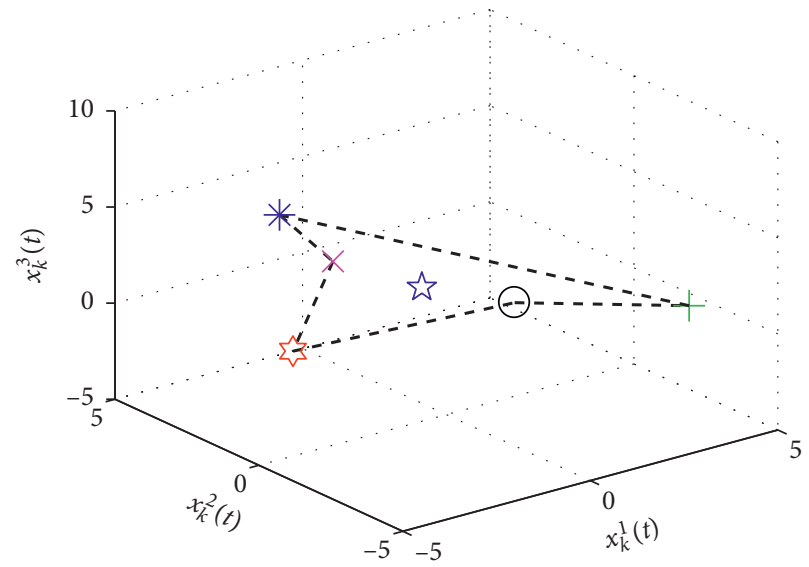

(a)

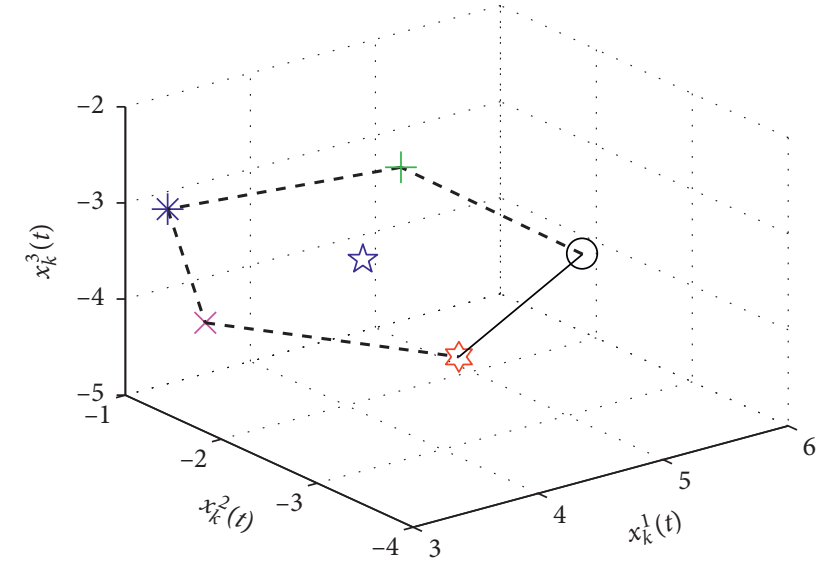

(b)

Figure 3: Continued. 


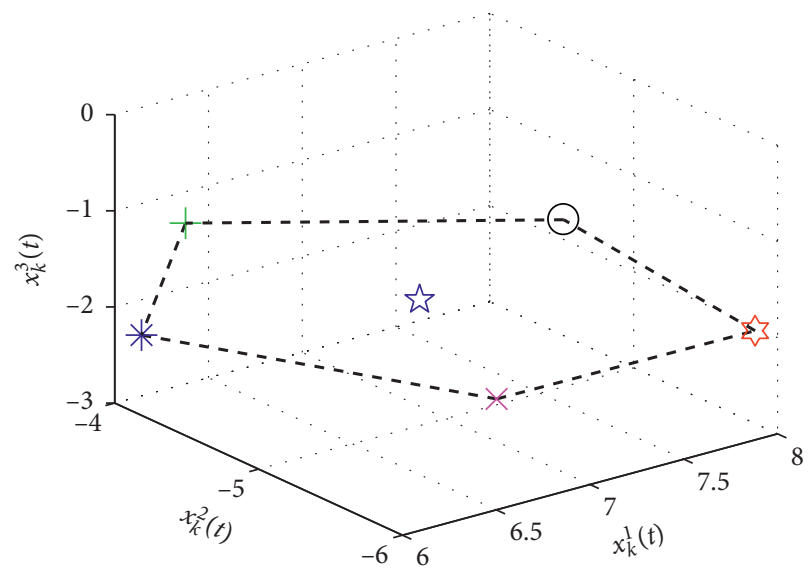

(c)

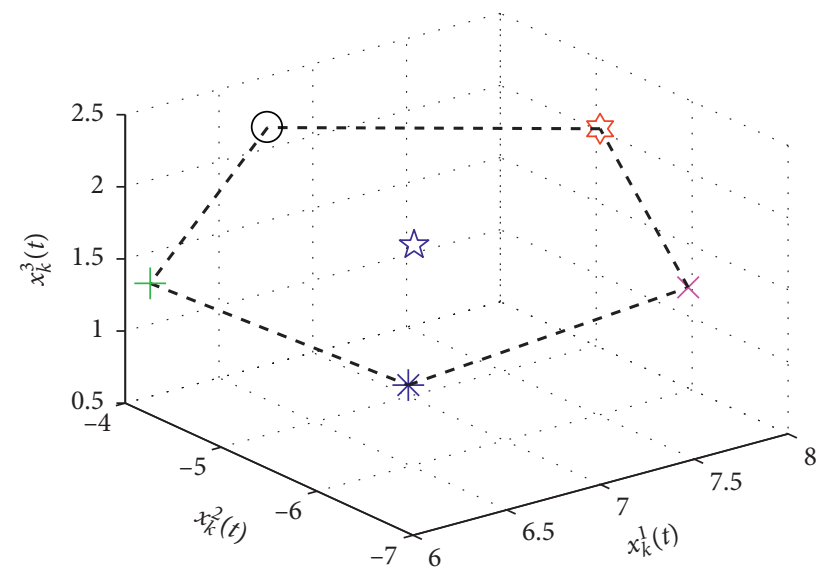

(d)

Figure 3: State snapshots of all the agents at different times: (a) $t=0 \mathrm{~s}$, (b) $t=8 \mathrm{~s}$, (c) $t=9 \mathrm{~s}$, and (d) $t=10 \mathrm{~s}$.

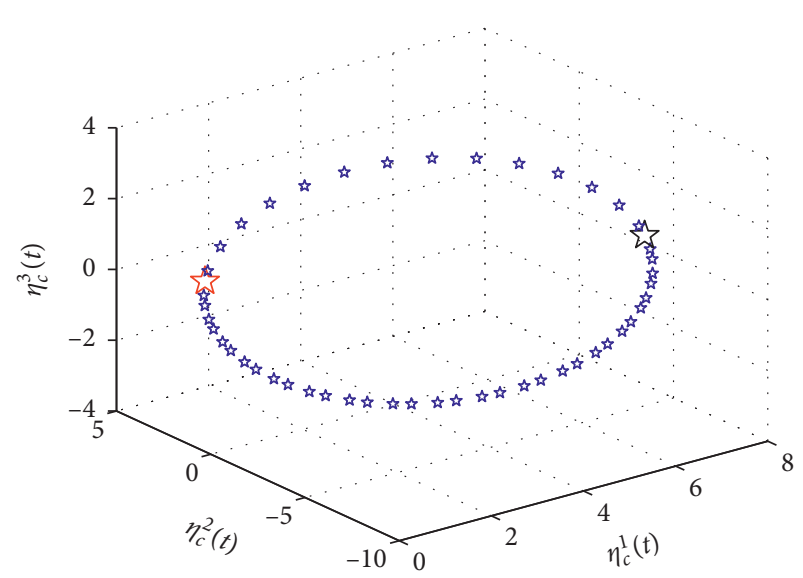

FIgURE 4: Movement track of the formation center function.

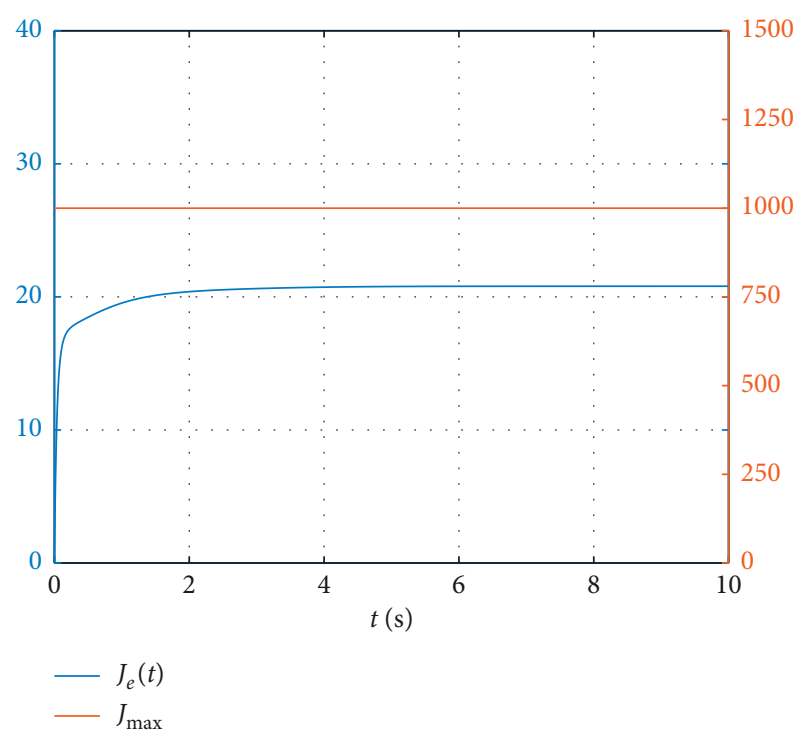

Figure 5: Practical energy consumption and the limited budget.

\section{Conclusions}

For high-order linear swarm systems with fixed communication topologies, a new formation control protocol with an energy integral term was proposed by using the state errors and the formation function errors among neighboring agents, which can guarantee that the practical energy assumption is less than the whole energy budget. Furthermore, by an orthonormal transformation associated with the Laplacian matrix of the fixed communication topology, limited-budget formation control problems were converted into asymptotic stability ones, and sufficient conditions for swam systems to achieve limitedbudget formation were proposed, where the relationship between the matrix variable and the limited budget was constructed on the basis of the specific structure feature of the orthonormal transformation matrix. In particular, those criteria are scalable and checkable since their constraints are independent of the number of agents and can be converted into linear matrix inequalities. Moreover, an explicit expression of the formation center function was presented, which contains two independent parts associated with the formation functions and the cooperative states, but it is independent of the variances of the formation functions.

The further works will focus on extending the current results to the swarm systems with heterogeneous dynamics, and the fixed topology in this paper will be changed to the switching connected topologies and the jointly switching topologies.

\section{Data Availability}

The data used to support the findings of the study are available in Section 4 of this paper.

\section{Conflicts of Interest}

The authors declare no conflicts of interest. 


\section{Authors' Contributions}

Hongtao Dang and Le Wang conceptualized the study and prepared the original draft; Hongtao Dang investigated the data and was responsible for methodology and funding acquisition and involved in project administration; Yan Zhang validated the data; Jianye Yang performed formal analysis; Yan Zhang and Jianye Yang reviewed and edited the manuscript; Le Wang supervised the study. All authors have read and agreed to the published version of the manuscript.

\section{Acknowledgments}

This research was funded by the Key Research and Development Program of Shaanxi (no. 2019GY-025), also funded by the National Natural Science Foundation of China under Grants 61867005.

\section{References}

[1] J. Zhou, X. Wu, W. Yu, M. Small, and J.-A. Lu, "Flocking of multi-agent dynamical systems based on pseudo-leader mechanism," Systems \& Control Letters, vol. 61, no. 1, pp. 195-202, 2012.

[2] R. Olfati-Saber, "Flocking for multi-agent dynamic systems: algorithms and theory," IEEE Transactions on Automatic Control, vol. 51, no. 3, pp. 401-420, 2006.

[3] J. Xi, C. Wang, H. Liu, and L. Wang, "Completely distributed guaranteed-performance consensualization for high-order multiagent systems with switching topologies," IEEE Transactions on Systems, Man, and Cybernetics: Systems, vol. 49, no. 7, pp. 1338-1348, 2019.

[4] J. Qu, Z. Ji, C. Lin, and H. Yu, "Fast consensus seeking on networks with antagonistic interactions," Complexity, vol. 78, 2018.

[5] J. Sun, Z. Geng, Y. Lv, Z. Li, and Z. Ding, "Distributed adaptive consensus disturbance rejection for multi-agent systems on directed graphs," IEEE Transactions on Control of Network Systems, vol. 5, no. 1, pp. 202-212, 2018.

[6] J. Xi, C. Wang, X. Yang, and B. Yang, "Limited-budget output consensus for descriptor multiagent systems with energy constraints," IEEE Transactions on Cybernetics, vol. 1, 2020.

[7] Y. Zhang, H. Li, J. Sun, and W. He, "Cooperative adaptive event-triggered control for multiagent systems with actuator failures," IEEE Transactions on Systems, Man, and Cybernetics: Systems, vol. 49, no. 9, pp. 1759-1768, 2019.

[8] J. Xi, L. Wang, J. Zheng, and X. Yang, "Energy-constraint formation for multiagent systems with switching interaction topologies," IEEE Transactions on Circuits and Systems I: Regular Papers, vol. 67, no. 6, pp. 2442-2454, 2020.

[9] Y. Zhu, S. Li, J. Ma, and Y. Zheng, "Bipartite consensus in networks of agents with antagonistic interactions and quantization," IEEE Transactions on Circuits and Systems II: Express Briefs, vol. 65, no. 12, pp. 2012-2016, 2018.

[10] Y. Zheng, Q. Zhao, J. Ma, and L. Wang, "Second-order consensus of hybrid multi-agent systems," Systems \& Control Letters, vol. 125, pp. 51-58, 2019.

[11] Y. Zheng, J. Ma, and L. Wang, "Consensus of hybrid multiagent systems," IEEE Transactions on Neural Networks and Learning Systems, vol. 29, no. 4, pp. 1359-1365, 2018.

[12] L. Consolini, F. Morbidi, D. Prattichizzo, and M. Tosques, "Leader-follower formation control of nonholonomic mobile robots with input constraints," Automatica, vol. 44, no. 5, pp. 1343-1349, 2008.

[13] K.-K. Oh and H.-S. Ahn, "Formation control of mobile agents based on distributed position estimation," IEEE Transactions on Automatic Control, vol. 58, no. 3, pp. 737-742, 2013.

[14] H. Liu, T. Ma, F. L. Lewis, and Y. Wan, "Robust formation control for multiple quadrotors with nonlinearities and disturbances," IEEE Transactions on Cybernetics, vol. 50, no. 4, pp. 1362-1371, 2020.

[15] Z. Ji, H. Lin, S. Cao, Q. Qi, and H. Ma, "The complexity in complete graphic characterizations of multiagent controllability," IEEE Transactions on Cybernetics, vol. 50, 2020.

[16] L. Mo and S. Guo, "Consensus of linear multi-agent systems with persistent disturbances via distributed output feedback," Journal of Systems Science and Complexity, vol. 32, no. 3, pp. 835-845, 2019.

[17] S. Liu, Z. Ji, and H. Ma, "Jordan form-based algebraic conditions for controllability of multiagent systems under directed graphs," Complexity, vol. 2020, 2020.

[18] X.-G. Guo, J.-L. Wang, F. Liao, and D. Wang, "Quantized \$H_ $\{\backslash$ infty\}\$ consensus of multiagent systems with quantization mismatch under switching weighted topologies," IEEE Transactions on Control of Network Systems, vol. 4, no. 2, pp. 202-212, 2017.

[19] Z.-Y. Tan, N. Cai, J. Zhou, and S.-G. Zhang, "On performance of peer review for academic journals: analysis based on distributed parallel system," IEEE Access, vol. 7, pp. 19024-19032, 2019.

[20] W. Ren, "Consensus strategies for cooperative control of vehicle formations," IET Control Theory \& Applications, vol. 1, no. 2, pp. 505-512, 2007.

[21] J. A. Fax and R. M. Murray, "Information flow and cooperative control of vehicle formations," IEEE Transactions on Automatic Control, vol. 49, no. 9, pp. 1465-1476, 2004.

[22] H. Du, G. Wen, Y. Cheng, Y. He, and R. Jia, "Distributed finite-time cooperative control of multiple high-order nonholonomic mobile robots," IEEE Transactions on Neural Networks and Learning Systems, vol. 28, no. 12, pp. 29983006, 2016.

[23] W. Qin, Z. Liu, and Z. Chen, "A novel observer-based formation for nonlinear multi-agent systems with time delay and intermittent communication," Nonlinear Dynamics, vol. 79, no. 3, pp. 1651-1664, 2015.

[24] L. Brinon-Arranz, A. Seuret, and C. Canudas-de-Wit, "Cooperative control design for time-varying formations of multiagent systems," IEEE Transactions on Automatic Control, vol. 59, no. 8, pp. 2283-2288, 2014.

[25] R. Rahimi, F. Abdollahi, and K. Naqshi, "Time-varying formation control of a collaborative heterogeneous multi agent system," Robotics and Autonomous Systems, vol. 62, no. 12, pp. 1799-1805, 2014.

[26] X. Dong and G. Hu, "Time-varying formation control for general linear multi-agent systems with switching directed topologies," Automatica, vol. 73, no. 73, pp. 47-55, 2016.

[27] F. Xiao, L. Wang, J. Chen, and Y. Gao, "Finite-time formation control for multi-agent systems," Automatica, vol. 45, no. 11, pp. 2605-2611, 2009.

[28] X. W. Dong, Y. Zhou, Z. Ren, and Y. S. Zhong, "Time-varying formation tracking for second-order multi-agent systems subjected to switching topologies with application to quadrotor formation flying," IEEE Transactions on Industrial Electronics, vol. 64, no. 6, pp. 5014-5024, 2016.

[29] X. Dong and G. Hu, "Time-varying formation tracking for linear multiagent systems with multiple leaders," IEEE 
Transactions on Automatic Control, vol. 62, no. 7, pp. 36583664, 2017.

[30] J. Xi, Z. Fan, H. Liu, and T. Zheng, "Guaranteed-cost consensus for multiagent networks with Lipschitz nonlinear dynamics and switching topologies," International Journal of Robust and Nonlinear Control, vol. 28, no. 7, pp. 2841-2852, 2018.

[31] L. Wang, J. Xi, M. He, and G. Liu, "Robust time-varying formation design for multiagent systems with disturbances: extended-state-observer method," International Journal of Robust and Nonlinear Control, vol. 30, no. 7, pp. 2796-2808, 2020.

[32] J. Yu, X. Dong, Q. Li, and Z. Ren, "Robust $\$\left\{\mathrm{H}_{-}\right.$linfty $\}$ guaranteed cost time-varying formation tracking for highorder multiagent systems with time-varying delays," IEEE Transactions on Systems, Man, and Cybernetics: Systems, vol. 50, no. 4, pp. 1465-1475, 2020.

[33] C. Godsil and G. Royal, Algebraic Graph Theory, SpringerVerlag, New York, NY, USA, 2001.

[34] S. Boyd, L. E. Ghaoui, E. Feron, and V. Balakrishnan, Linear Matrix Inequalities in System and Control Theory, SIAM, Philadelphia, PA, USA, 1994

[35] P. Gahinet, A. Nemirovskii, A. J. Laub, and M. Chilali, LMI Control Toolbox User's Guide, The Math Works, Natick, MA, USA, 1995.

[36] A. Berman and X. D. Zhang, "Lower bounds for the eigenvalues of Laplacian matrices," Linear Algebra and its Applications, vol. 316, no. 1-3, pp. 13-20, 2000.

[37] R. A. Horn and C. A. Johnson, Matrix Analysis, Cambridge University, London, UK, 1990. 